\title{
The Analytical Review of the Condition of Heavy Class Military and Dual- Purpose Unmanned Ground Vehicle
}

\author{
Aleksandr Blokhin $^{1, a}$, Alla Koshurina ${ }^{1, b}$, Maxim Krasheninnikov ${ }^{1, c}$, Roman Dorofeev $^{1, d}$ \\ ${ }^{1}$ Transport Scientific and Educational Centre, Nizhny Novgorod State Technical University,named after R.E. Alekseev, Russia
}

\begin{abstract}
The purpose of this article is the evaluation of the actual condition of heavy (weight more than $700 \mathrm{~kg}$ ) military robotics and dual-purpose robotics in the world. The extensive review of the world market of heavy class military unmanned ground vehicle was made. All reviewed robots are used at present time or exist like prototypes. All robots were systematized by most important technical characteristics. In the closing of article the analysis of the reviewed heavy class dual purpose UGVs are presented. Based on the analysis the conclusion about actual condition of the heavy military robotics and dual-purpose robotics was made. Also the most promising ways and tendencies of development are representeds.
\end{abstract}

\section{Introduction}

By the end of 2004, there were 150 robots in US military operations in Iraq; a year later there were 2,400; by the end of 2008, there were about 12,000 robots in military operations in Iraq [1], wherein only 5331 of them were unmanned aerial vehicles [2].

At the current rate, the US Army is expected to shrink from 540,000 people down to 490,000 to the end of 2015 year and shrink to 420,000 by 2019 , as was said in DefenseNews [3]. Gen. Robert Cone, head of the Army's Training and Doctrine Command, offered some details about the slim-down plans. Robert Cone added that the US Army is studying the possibility of reducing the size of a brigade from 4,000 soldiers to 3,000 in the coming years. To keep things just as effective while reducing manpower, the Army will bring in more unmanned power, in the form of robots [4].

Besides United States, other countries embed robots in their Armies as well and do it quite actively. For example, the Ministry of Defence of the Russian Federation announced about intention to develop robotic systems and to use them for military purposes. According to the ministry, the robots will begin actively "storming" the army in $2017-2018$, and by 2025 approximately $30 \%$ of all military technology in the Russian Armed Forces will consist of robotic hardware [5].

\section{Review of Heavy Class UGVs}

Heavy class robots (weigh more than $700 \mathrm{~kg}$ ) have most practical interest, because such class of unmanned ground vehicles (UGV) can give real tactical advantages on a battlefield in contrast of light class UGV, which are not able to have enough firepower for serious battles and carry enough useful payload.

Our scientific collective have made the review of military and dual-purpose heavy class unmanned ground vehicles among various countries. More than 35 samples of such robots were found. Some of them was built and used in real battles; some - are on different stages of introduction (prototype, experimental sample, taken to weaponry, are using today and etc.)

Heavy class UGVs (Table 1) which stand out by their technical characteristics among other robots are presented in this article. Main technical characteristics are: universality, range and operating time, autonomy, allterrain abilities.

\footnotetext{
$\overline{\mathrm{a}}$ a.n.blokhin@gmail.com ${ }^{\mathrm{b}}$ allakoshurina@yandex.ru ${ }^{\mathrm{c}}$ maxim.krasheninnikov@mail.ru ${ }^{\mathrm{d}}$ blonde-o@yandex.ru
} 
Table 1. The comparison table of heavy class UGVs

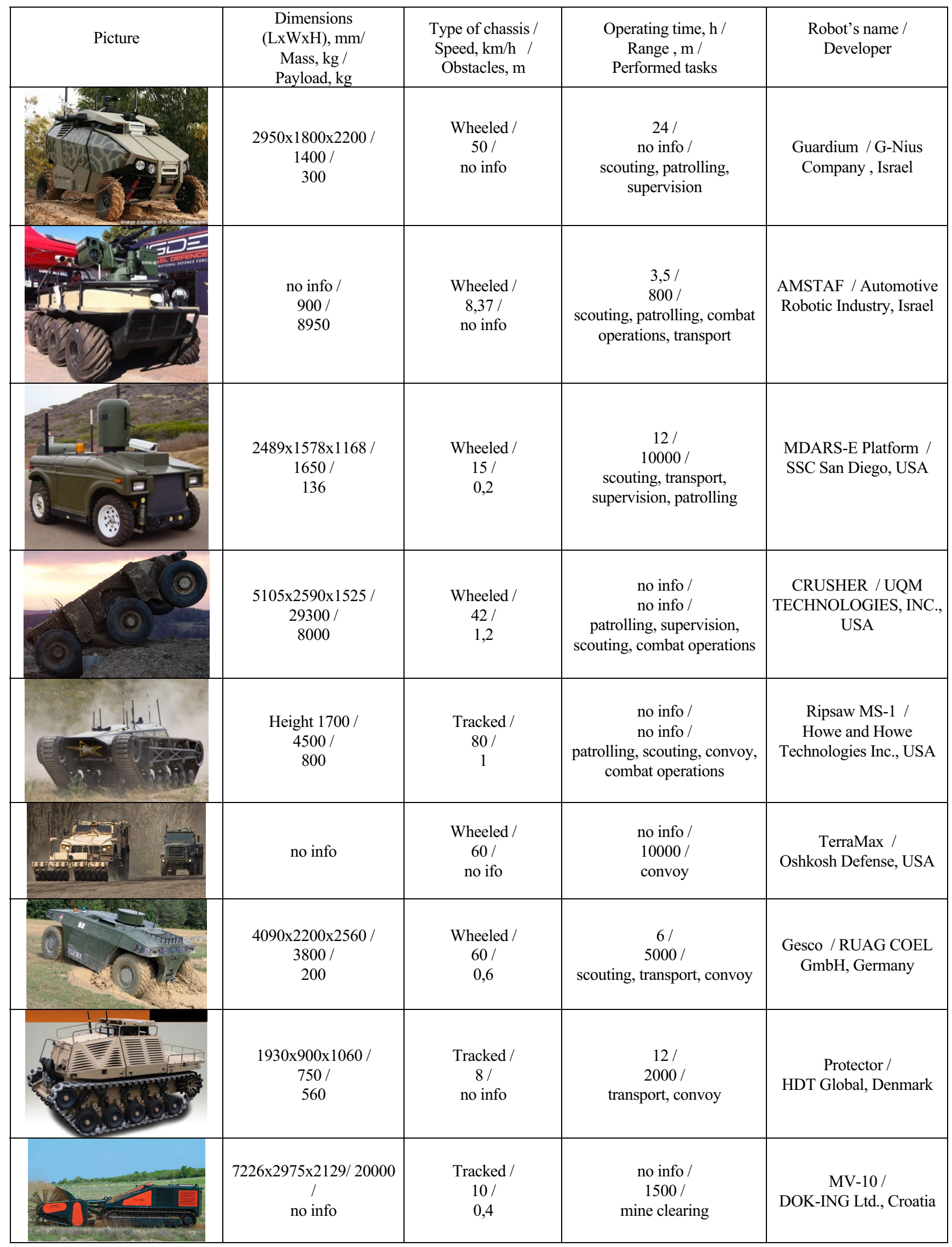




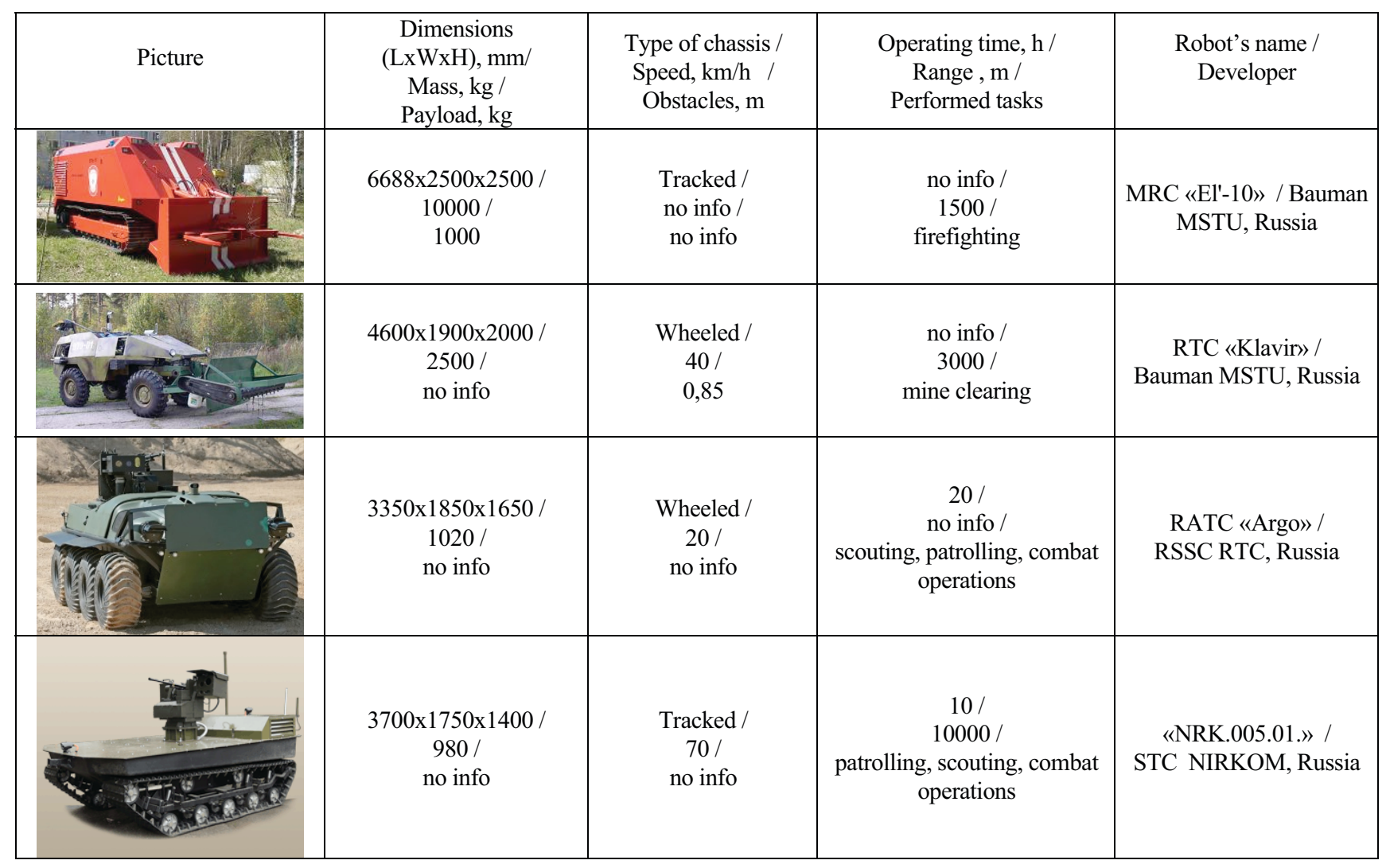

\section{Analysis of Heavy Class UGVs}

Analysis shows that a tracked chassis is a most often used type of chassis for heavy UGVs (Fig.1). Also results of the analysis show that heavy class UGVs are used for scouting and patrolling (Fig. 2).

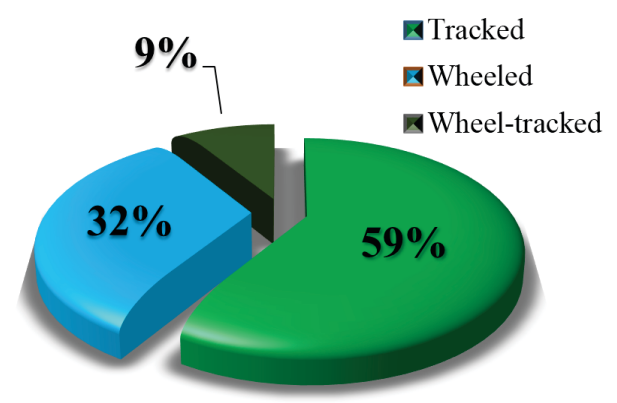

Figure 1. Types of chassis

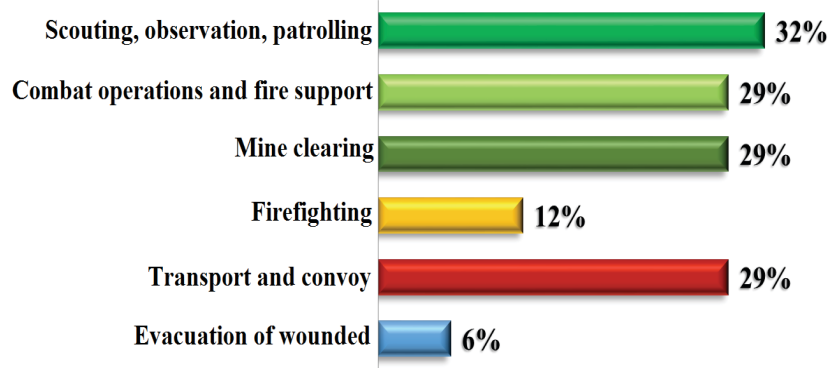

Figure 2. Performed tasks for heavy UGVs

The data in Table 1 shows that the most universal and functional robots are:
- Avantguard MK, AMSTAF, Israel;

- CRUSHER, Gladiator, Ripsaw MS-1, USA;

RATC «Argo», «NRK.005.01.», Russia;

Also robots, which have great range:

- MDARS-E Platform, TerraMax, USA;

- Gesco, Germany;

- RTC «Klavir», «NRK.005.01.», Russia.

The main advantage of Ripsaw MS1 UGV is the ability to move with high speed $(80 \mathrm{~km} / \mathrm{h})$. No one of heavy class unmanned ground vehicles can reach such speed. Developers of TerraMax convoy could realize a prototype of group interaction of unmanned ground vehicles. The column of five unmanned vehicles can move autonomous. There is an exchange of information about condition of environment, location in local and global coordinate systems between vehicles in the column. Uninhabited vehicles decide to bypass obstacles and choose a trajectory of movement by himself [6].

\section{Development Trends and Concepts}

Among developers, there is a trend to optimize and develop control algorithms, which let to unmanned vehicles, choose a trajectory, a regime of movement and perform missions without human-operator.

For unmanned ground vehicles to be affective in the modern battlespace, they must be able to work autonomously and together in teams. They will need to share data and be able to run semi-autonomously if the tele-robotic connection is broken. These unmanned vehicles like the modern UAVs will need to go into a safety mode and protect themselves and use artificially 
intelligent software and collaborative connections with other robots on the battlefield to continue the mission [7, 8].

Advantages of the group interaction can be in:

plenty of performed missions, because a group of robots may include robots with different abilities and combinations of robots abilities will let to complete different missions;

- the reliability of a group of robots always higher than the reliability of one robot, because "death" of a one robot didn't mean "death" of the group and it let to complete a mission successfully.

- a large coverage area and a better information awareness is as result. Undoubtedly, the plenty of robots can explore and control more territory than one robot;

- a tactical advantage in a battlefield. All advantages, which were called above, can give tactical advantage in a battle.

For "indirect contact" UGVs is necessary to increase the battery life to perform special operations (scouting, information gathering operations behind enemy lines), and also focus on the reduction of noise in the selection of engine.

One of the main trend for "assault" UGVs is the increasing of a payload. This trend can be applied also to supply vessel UGVs. The development of secure protocols of a information transfering and the formation of a unmistakable algorithm of enemy detecting are also extremely relevant for assault UGVs.

The trend for tele-operated UGVs (mine clearing, firefighter and rescue robots) is the increasing of a coverage area and using the wireless connection.

\section{Acknowledgements}

This work was carried out at the NSTU named after R.E. Alekseev, with financial support from the government in the face of the Russian Ministry of Education under the Federal Program "Research and development on priority directions of the scientific-technological complex of Russia for 2014-2020", the unique identifier of the project: RFMEFI57714X0105.

\section{References}

1. Information on http://www.brookings.edu/research/articles/2009/02/ winter-robots-singer

2. P.W. Singer: Joint Force Quarterly issue $52,1^{\text {st }}$ quarter 2009, p. 104-110

3. Information on http://www.defensenews.com/article/20140120/DEF REG02/301200035/US-Army-Studying-ReplacingThousands-Grunts-Robots

4. Information on http:/www.militarytimes.com/article/20140120/NE WS04/301200034/Army-studying-replacingthousands-grunts-robots

5. Information on http://www.telegraph.co.uk/sponsored/rbth/technolog y/11370393/universal-soldier-russia-militaryrobots.html

6. Information on http://oshkoshdefense.com/products/vehiclecomponents/terramax/

7. L. Winslow in: Unmanned Vehicle Robotic Warfare "Hide and Seek Strategies", (2007)

M. R. Blackburn, R. T. Laird, H. R. Everett: Unmanned Ground Vehicle (UGV) Lessons Learned, Technical Report 1869 (2001) 